\title{
Dynamic Depolarized Light Scattering by Oligo- and Poly $(\alpha$-methylstyrene)s in Dilute Solution
}

\author{
Hiroshi UEDA, Masashi OsA, Takenao YoshIZAKI, ${ }^{\dagger}$ and Hiromi YAMAKAWA \\ Department of Polymer Chemistry, Kyoto University, Katsura, Kyoto 615-8510, Japan
}

(Received July 30, 2004; Accepted September 9, 2004; Published January 15, 2005)

\begin{abstract}
The power spectrum $J_{\Gamma}$ of the excess depolarized component of scattered light was determined from frequency-domain dynamic depolarized light scattering measurements for 10 samples of atactic oligo- and poly $(\alpha-$ methylstyrene)s (a-P $\alpha \mathrm{MS}$ ), each with the fraction of racemic diads $f_{\mathrm{r}}=0.72$, in the range of weight-average degree of polymerization $x_{\mathrm{w}}$ from 2 to 67.1 in cyclohexane at $30.5^{\circ} \mathrm{C}(\Theta)$. It is found that, as in the cases of atactic polystyrene (a-PS), atactic poly(methyl methacrylate) (a-PMMA), and isotactic (i-) PMMA, $J_{\Gamma}$ may be well represented in terms of a single Lorentzian independently of $x_{\mathrm{w}}$, and that the relaxation time $\tau_{\Gamma}$ as defined as the reciprocal of the half-width at half-maximum of $J_{\Gamma}$ evaluated at infinite dilution increases with increasing $x_{\mathrm{w}}$ and levels off to its asymptotic value in the limit of $x_{\mathrm{W}} \rightarrow \infty$, being consistent with the theoretical prediction on the basis of the helical wormlike (HW) chain model. A comparison is made of the present data for $\tau_{\Gamma}$ with the HW theory, and it is shown that the theory may explain satisfactorily the data in the range of $x_{\mathrm{w}} \gtrsim 10$, although semiquantitatively. For $x_{\mathrm{w}} \lesssim 10$, the rigid sphere model having the radius equal to the apparent root-mean-square radius of gyration of the HW chain may give a good explanation of $\tau_{\Gamma}$. From a comparison of the present results for $\tau_{\Gamma}$ for a-P $\alpha$ MS with previous ones for a-PS and a- and i-PMMAs, it is shown that there is good correlation between static and dynamic chain stiffness, the latter being defined as the ratio of the relaxation time associated with the local motion of the long chain to that of its isolated repeat unit (monomer), as predicted by the HW theory. [DOI $10.1295 /$ polymj.37.7]

KEY WORDS Poly $(\alpha$-methylstyrene) / Dynamic Depolarized Light Scattering / Spectrum of Depolarized Scattered Component / Helical Wormlike Chain / Dynamic Chain Stiffness /
\end{abstract}

In a new framework of polymer solution science on the basis of the helical wormlike (HW) chain model, ${ }^{1,2}$ we have recently made a series of experimental studies $^{3-6}$ of dilute solution behavior of atactic oligoand poly $(\alpha$-methylstyrene)s (a-P $\alpha \mathrm{MS})$ in the unperturbed $\Theta$ state. Specifically, we have determined the mean-square radius of gyration $\left\langle S^{2}\right\rangle,{ }^{3}$ mean-square optical anisotropy $\left\langle\Gamma^{2}\right\rangle,{ }^{4}$ scattering function, ${ }^{5}$ and steady-state transport coefficients ${ }^{6}$ in cyclohexane at $30.5^{\circ} \mathrm{C}(\Theta),{ }^{3}$ using well-fractionated a-P $\alpha$ MS samples with the fraction of racemic diads $f_{\mathrm{r}}$ regulated to be $0.72 \pm 0.01 .^{7}$ The results obtained have been analyzed to show that the a-P $\alpha$ MS chain has rather large chain stiffness and strong helical nature like the atactic poly(methyl methacrylate) (a-PMMA) chain with $f_{\mathrm{r}}=0.79 .{ }^{1,8}$ Here, by the term "helical nature," we mean that the chain tends to retain large and clearly distinguishable helical portions in dilute solution. ${ }^{1}$

In the present and following ${ }^{9}$ papers, we proceed to make a study of dynamical properties of a-P $\alpha$ MS and its oligomers at $\Theta$. We have already investigated as those properties the spectrum $J_{\Gamma}$ of the (excess) depolarized scattered component, spin-lattice relaxation time $T_{1}$, and nuclear Overhauser enhancement NOE for atactic polystyrene (a-PS), ${ }^{10}$ a-PMMA,${ }^{11}$ and isotactic (i-) PMMA. ${ }^{12}$ The conclusions derived from an analysis of experimental data for them within the framework of the dynamic theory ${ }^{1,13-15}$ of the (unperturbed) $\mathrm{HW}$ chain are the following. First, $J_{\Gamma}$ may be well represented in terms of a single Lorentzian, and the relaxation time $\tau_{\Gamma}$ defined by the reciprocal of the half-width at half-maximum (HWHM) of $J_{\Gamma}$ increases with increasing weight-average degree of polymerization $x_{\mathrm{w}}$ and levels off to its asymptotic value in the limit of $x_{\mathrm{w}} \rightarrow \infty$, the results being consistent with the dynamic HW theory prediction. ${ }^{1,13}$ Second, $T_{1}$ and NOE are closely correlated with $\tau_{\Gamma}$. Third, the dynamic HW theories ${ }^{1,13-15}$ may explain semiquantitatively the dependences on $x_{\mathrm{w}}$ of these three properties for $x_{\mathrm{w}} \gtrsim 10$ except for NOE for a-PS, while the rigid sphere model with a single, proper relaxation time is valid for them for smaller $x_{\mathrm{w}}$. Fourth, as far as the above three polymers are concerned, there is good correlation between dynamic and static chain stiffness, as predicted by the HW theory. ${ }^{1,15}$ Thus the main purpose of the present and following papers is to examine whether the above conclusions are also valid for a-P $\alpha$ MS.

In the present paper, we carry out frequency-

${ }^{\dagger}$ To whom correspondence should be addressed (E-mail: yoshizaki@molsci.polym.kyoto-u.ac.jp). 
domain dynamic depolarized light scattering (LS) measurements to determine $J_{\Gamma}$ for a-P $\alpha \mathrm{MS}$ and its oligomers in cyclohexane at $30.5^{\circ} \mathrm{C}(\Theta)$ by the use of a Fabry-Perot (FP) interferometer in the manner established in previous papers, ${ }^{10-12}$ and then examine the behavior of $\tau_{\Gamma}$ as a function of $x_{\mathrm{w}}$. Results of nuclear magnetic relaxation measurements and their analysis are reported in the following paper. ${ }^{9}$

\section{EXPERIMENTAL}

\section{Materials}

All the a-P $\alpha$ MS samples used in this work are the same as those used in the previous study of $\left\langle\Gamma^{2}\right\rangle,{ }^{4}$ i.e., fractions separated by preparative gel permeation chromatography (GPC) or fractional precipitation from the original samples prepared by living anionic polymerization. ${ }^{3,7} \mathrm{We}$ note that the initiating chain end of each polymerized sample is a sec-butyl group and the other end is a hydrogen atom.

The values of the weight-average molecular weight $M_{\mathrm{w}}$ determined from ${ }^{1} \mathrm{H}$ and ${ }^{13} \mathrm{C}$ NMR spectra, by analytical GPC, or from (static) LS measurements (in cyclohexane at $30.5^{\circ} \mathrm{C}$ ), $x_{\mathrm{w}}$ estimated from $M_{\mathrm{w}}$, the ratio of $M_{\mathrm{w}}$ to the number-average molecular weight $M_{\mathrm{n}}$ determined by analytical GPC, ${ }^{3,6}$ and $f_{\mathrm{r}}$ determined from ${ }^{1} \mathrm{H}$ and ${ }^{13} \mathrm{C}$ NMR spectra ${ }^{3,7}$ are given in Table I. Although $f_{\mathrm{r}}$ of the samples OAMS4OAMS10 could not be determined because of the complexity of their ${ }^{1} \mathrm{H}$ NMR spectra, they may be regarded as having almost the same value of $f_{\mathrm{r}}(0.71 \lesssim$ $\left.f_{\mathrm{r}} \lesssim 0.73\right)$ as OAMS3 or OAMS13 with $M_{\mathrm{w}}=1.60 \times$ $10^{3}$ (which is not used in this work), since the samples OAMS3-OAMS13 are fractions from one original sample. ${ }^{7}$ As seen from the values of $f_{\mathrm{r}}$, all the samples except OAMS2 have the fixed stereochemical composition $f_{\mathrm{r}}=0.72 \pm 0.01$. As previously ${ }^{6}$ mentioned, the difference in $f_{\mathrm{r}}$ between the sample OAMS2 and the others may be regarded as arising from that in reaction mechanism; i.e., $f_{\mathrm{r}}$ of the former is determined by the

Table I. Values of $M_{\mathrm{w}}, x_{\mathrm{w}}, M_{\mathrm{w}} / M_{\mathrm{n}}$, and $f_{\mathrm{r}}$ for atactic oligo- and poly $(\alpha$-methylstyrene)s

\begin{tabular}{ccccc}
\hline Sample & $M_{\mathrm{w}}$ & $x_{\mathrm{w}}$ & $M_{\mathrm{w}} / M_{\mathrm{n}}$ & $f_{\mathrm{r}}$ \\
\hline OAMS2 & $2.94 \times 10^{2}$ & 2 & 1 & 0.56 \\
OAMS3 & $4.12 \times 10^{2}$ & 3 & 1 & 0.73 \\
OAMS4 & $5.30 \times 10^{2}$ & 4 & $<1.01$ & \\
OAMS5 & $6.48 \times 10^{2}$ & 5 & $<1.01$ & \\
OAMS6 & $7.66 \times 10^{2}$ & 6 & $<1.01$ & \\
OAMS8 & $1.04 \times 10^{3}$ & 8.29 & 1.01 & \\
OAMS10 & $1.27 \times 10^{3}$ & 10.3 & 1.01 & \\
OAMS19 & $2.27 \times 10^{3}$ & 18.7 & 1.07 & 0.72 \\
OAMS38 & $4.57 \times 10^{3}$ & 38.2 & 1.07 & 0.72 \\
OAMS67 & $7.97 \times 10^{3}$ & 67.1 & 1.04 & 0.72 \\
\hline
\end{tabular}

termination reaction with methanol, while those of the latter are determined (mainly) by the propagation reaction. As seen from the values of $M_{\mathrm{w}} / M_{\mathrm{n}}$, all the samples are very narrow in molecular weight distribution.

The solvent cyclohexane used for dynamic depolarized LS measurements was purified according to a standard procedure prior to use.

\section{Dynamic Depolarized Light Scattering}

The photometer used for all dynamic depolarized LS measurements is the same as that used in the previous studies, ${ }^{4,10-12,16,17}$ i.e., a Brookhaven Instruments Model BI-200SM goniometer with a minor modification of its light source part and with a detector alignment previously assembled to incorporate an FP interferometer in it. It has been described in detail in a previous paper, ${ }^{16}$ and therefore we here only give its short sketch (see Figure 1 of ref 16).

Vertically polarized light of wavelength $488 \mathrm{~nm}$ from a Spectra-Physics Model 2020 argon ion laser equipped with a Model 583 temperature-stabilized etalon for single-frequency-mode operation was used as a light source. It was made highly vertically (v) polarized by passing through a Gran-Thompson (GT) prism with an extinction ratio smaller than $10^{-5}$. The scattered light was measured at a scattering angle of $90^{\circ}$. Its horizontal $(\mathrm{H})$ component, i.e., the depolarized (Hv) component, which was extracted from the total scattered light intensity by the use of the same GT prism as above, was analyzed with a Burleigh Instruments Model RC-110 FP interferometer equipped with a Model RC-670 pair of plane mirrors with a flatness of $\lambda / 200$ and a reflectivity of $97.5 \%$. The intensity of the Hv component filtered through the FP interferometer was measured by an EMI 9893B/350 photomultiplier (PM) tube. A pinhole of diameter $100 \mu \mathrm{m}$ was used as a spatial filter, which was placed between the interferometer and the PM tube. All the measurements were carried out by the single passing as in the previous studies. ${ }^{10-12}$

As before, ${ }^{10-12}$ the transmittance $T(\omega)$ of the FP interferometer was determined by measuring the polarized component of the light intensity scattered from a solution of an a-PS sample with $M_{\mathrm{w}}=7.32 \times 10^{5}$ in methyl ethyl ketone at the concentration $c \simeq 1 \times$ $10^{-3} \mathrm{~g} / \mathrm{cm}^{3}$ and at $30.5^{\circ} \mathrm{C}$ following the procedure of Ouano and Pecora. ${ }^{18}$

In the present study, the free spectral range (FSR) of the interferometer (i.e., the distance between the mirrors) was changed from $4.71 \times 10^{10}$ to $8.66 \times$ $10^{11} \mathrm{rad} / \mathrm{s}$ depending on the width of $J_{\Gamma}$ in order to attain an appropriate resolution. As in the previous studies, ${ }^{10-12}$ the value $\delta$ of FSR for each setting was determined by the use of the ${ }^{4} \mathrm{He}$ hyperfine structure 
interval of $1.0 \mathrm{~cm}^{-1}$ at $587.5 \mathrm{~nm}$ associated with the $2^{3} \mathrm{P}-3^{3} \mathrm{D}$ transition for $3 \times 10^{11} \lesssim \delta \lesssim 9 \times 10^{11} \mathrm{rad} / \mathrm{s}$ and of the Brillouin shift $\Delta \omega_{\mathrm{B}}$ of $3.53 \times 10^{10} \mathrm{rad} / \mathrm{s}$ in the Rayleigh-Brillouin spectrum of pure benzene at $25.0^{\circ} \mathrm{C}$ at $1 \mathrm{~atm}$ for $10^{11} \lesssim \delta \lesssim 3 \times 10^{11} \mathrm{rad} / \mathrm{s}$. For $\delta \lesssim 10^{11} \mathrm{rad} / \mathrm{s}$, the distance between the mirrors was directly measured.

Measurements were carried out on solutions of all the samples in cyclohexane at $30.5^{\circ} \mathrm{C}(\Theta)$. The most concentrated solution of each sample in cyclohexane was prepared gravimetrically and made homogeneous by continuous stirring at $c a .50^{\circ} \mathrm{C}$ for $1-2 \mathrm{~d}$. This solution and the solvent were optically purified by filtration through a Teflon membrane of pore size 0.1 or $0.45 \mu \mathrm{m}$. The solutions of lower concentrations were obtained by successive dilution. The weight concentrations of the test solutions were converted to the solute mass concentrations $c$ (in $\mathrm{g} / \mathrm{cm}^{3}$ ) by the use of the densities of the solutions.

Before and after each measurement on the solution or solvent, the transmittance of the FP interferometer was determined in the manner as described above. In the present measurements, the finesse could be kept at 30-60 during a single measurement, which took $c a$. $40 \mathrm{~min}$.

\section{RESULTS}

In each frequency-domain measurement of the intensity $I_{\mathrm{Hv}}$ of the depolarized (Hv) component of the light scattered from a test solution by the use of the FP interferometer, an apparent spectrum $I_{\mathrm{Hv}, \mathrm{ap}}\left(\Delta \omega_{\mathrm{ap}}\right)$ is observed as a function of the difference $\Delta \omega_{\text {ap }}$ between the angular frequencies $\omega$ of the peak of the transmittance $T(\omega)$ of the FP interferometer and of the incident light. The spectrum $I_{\mathrm{Hv}, \mathrm{ap}}\left(\Delta \omega_{\mathrm{ap}}\right)$ so observed may be represented by a convolution integral of $T(\omega)$ and the true spectrum $I_{\mathrm{Hv}}(\Delta \omega)$ as a function of the difference $\Delta \omega$ between $\omega$ 's of the scattered and incident light waves, i.e., the integral of $T(\Delta \omega-$ $\left.\Delta \omega_{\mathrm{ap}}\right) I_{\mathrm{Hv}}(\Delta \omega)$ over $\Delta \omega .^{10,19}$ Further, $I_{\mathrm{Hv}}(\Delta \omega)$ is in general composed of the intrinsic depolarized spectrum $J_{\Gamma}(\Delta \omega)$ due to the solute a-P $\alpha$ MS and that due to the solvent molecule. Fortunately, in the present case, the optical anisotropy of the solvent cyclohexane is very small compared to that of the solute a-P $\alpha \mathrm{MS}$ as in the previous case of a-PS in cyclohexane or carbon tetrachloride, ${ }^{10}$ so that $I_{\mathrm{Hv}}(\Delta \omega)$ may be equated to $J_{\Gamma}(\Delta \omega)$. Then, assuming that $J_{\Gamma}\left(I_{\mathrm{Hv}}\right.$ in the present case) may be represented by a linear combination of several (practically six) Lorentzians and using $T(\omega)$ experimentally determined, the coefficient (weight) and the HWHM of each Lorentzian may be determined by numerical deconvolution of $I_{\mathrm{Hv}, \mathrm{ap}} \cdot{ }^{10-12}$

As in the cases of a-PS ${ }^{10}$ and a- and i-PMMAs, ${ }^{11,12}$ the HWHMs of the six Lorentzians so determined for each test solution were found to be the same, indicating that $J_{\Gamma}$ may be fitted by a single Lorentzian. We then determine the (apparent) relaxation time $\tau_{\Gamma}$ defined by the reciprocal of the HWHM of $J_{\Gamma}$ thus determined, which is just the HWHM of the single Lorentzian in the present case. Figure 1 shows plots of $\tau_{\Gamma}$ against $c$ for all a-P $\alpha$ MS samples in cyclohexane at $30.5^{\circ} \mathrm{C}(\Theta)$. Each plot follows a straight line and the value of $\tau_{\Gamma}$ at infinite dilution may be determined from its intercept.

The values of $\tau_{\Gamma}$ so obtained at infinite dilution are given in Table II along with those of the reduced relaxation time $k_{\mathrm{B}} T \tau_{\Gamma} / \eta_{0}$ with $k_{\mathrm{B}}$ the Boltzmann constant, $T$ the absolute temperature, and $\eta_{0}$ the solvent

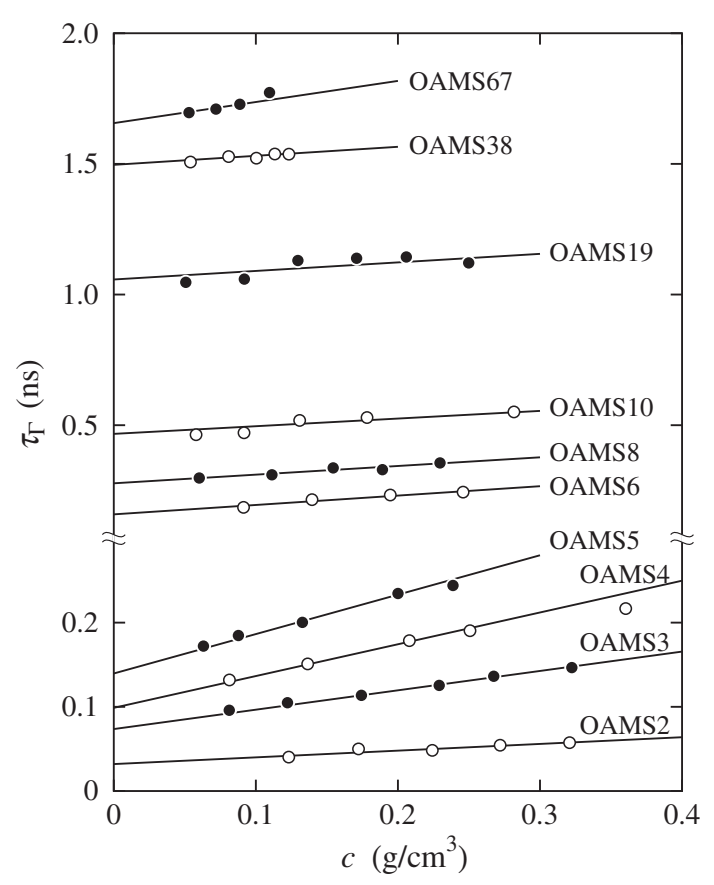

Figure 1. Plots of $\tau_{\Gamma}$ against $c$ for all a-P $\alpha$ MS samples in cyclohexane at $30.5^{\circ} \mathrm{C}(\Theta)$.

Table II. Values of $\tau_{\Gamma}$ and $k_{\mathrm{B}} T \tau_{\Gamma} / \eta_{0}$ for atactic oligo- and poly $(\alpha$-methylstyrene)s in cyclohexane at $30.5^{\circ} \mathrm{C}$ at infinite dilution

\begin{tabular}{lcc}
\hline Sample & $\begin{array}{c}\tau_{\Gamma} \\
(\mathrm{ns})\end{array}$ & $\begin{array}{c}k_{\mathrm{B}} T \tau_{\Gamma} / \eta_{0} \\
\left(\AA^{3}\right)\end{array}$ \\
\hline OAMS2 & $0.032_{0}$ & $1.6_{4} \times 10^{2}$ \\
OAMS3 & $0.073_{6}$ & $3.7_{7} \times 10^{2}$ \\
OAMS4 & $0.098_{8}$ & $5.0_{6} \times 10^{2}$ \\
OAMS5 & $0.14_{0}$ & $7.1_{5} \times 10^{2}$ \\
OAMS6 & $0.15_{9}$ & $8.1_{4} \times 10^{2}$ \\
OAMS8 & $0.27_{8}$ & $1.4_{2} \times 10^{3}$ \\
OAMS10 & $0.46_{7}$ & $2.3_{9} \times 10^{3}$ \\
OAMS19 & $1.0_{6}$ & $5.4_{2} \times 10^{3}$ \\
OAMS38 & $1.5_{0}$ & $7.6_{7} \times 10^{3}$ \\
OAMS67 & $1.6_{6}$ & $8.4_{9} \times 10^{3}$ \\
\hline
\end{tabular}


viscosity. The latter reduced values have been calculated from the former with the value $0.818 \mathrm{cP}$ of $\eta_{0}$ for cyclohexane at $30.5^{\circ} \mathrm{C}$. As discussed in the previous papers, ${ }^{10-12}$ the reduced relaxation time $k_{\mathrm{B}} T \tau_{\Gamma} / \eta_{0}$ must be independent of solvent condition if the motions of the small molecules or the motional units in the polymer chain may be described in the diffusion limit and if the local chain conformation does not depend on that condition. In the previous study of a$\mathrm{PS},{ }^{10}$ the values of $k_{\mathrm{B}} T \tau_{\Gamma} / \eta_{0}$ for cumene, which may be regarded as corresponding to the monomer (repeat unit) of a-P $\alpha$ MS as well as of a-PS, in cyclohexane at $34.5{ }^{\circ} \mathrm{C}$ and in carbon tetrachloride at $25.0^{\circ} \mathrm{C}$ have been found to agree well with each other, leading to the conclusion that the dynamics that governs $\tau_{\Gamma}$ may be described in the diffusion limit for solutions of a-PS in cyclohexane. This may also be the case with a-P $\alpha \mathrm{MS}$.

Figure 2 shows plots of $k_{\mathrm{B}} T \tau_{\Gamma} / \eta_{0}$ at infinite dilution against the logarithm of $x_{\mathrm{w}}$ for a-P $\alpha \mathrm{MS}$ in cyclohexane at $30.5^{\circ} \mathrm{C}(\Theta)$. In the figure, the solid and dashed curves represent the theoretical values for the HW model and the rigid sphere model, respectively, which are obtained and discussed in the next (DISCUSSION) section. As in the cases of a-PS ${ }^{10}$ and a- and i-PMMAs, ${ }^{11,12}$ the reduced relaxation time $k_{\mathrm{B}} T \tau_{\Gamma} / \eta_{0}$ increases with increasing $x_{\mathrm{w}}$ and seems to level off in the limit of $x_{\mathrm{w}} \rightarrow \infty$, the result being consistent with the HW theory. ${ }^{13}$ We have estimated its asymptotic value in this limit to be ca. $8.5 \times 10^{3} \AA^{3}$ from the observed value $8.4_{9} \times 10^{3} \AA^{3}$ of $k_{\mathrm{B}} T \tau_{\Gamma} / \eta_{0}$ for the sample OAMS67 with the largest $x_{\mathrm{w}}$, which is appreciably smaller than the corresponding value $2 \times 10^{4} \AA^{3}$ for a-PMMA, ${ }^{11}$ appreciably larger than the one $4 \times 10^{3} \AA^{3}$ for a-PS, ${ }^{10}$ and slightly larger than

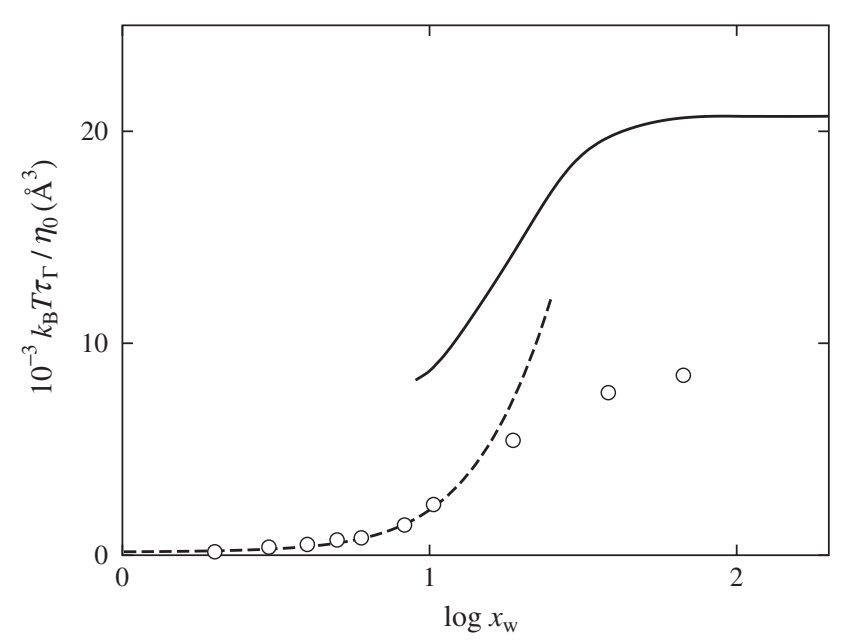

Figure 2. Plots of $k_{\mathrm{B}} T \tau_{\Gamma} / \eta_{0}$ at infinite dilution against the logarithm of $x_{\mathrm{w}}$ for a-P $\alpha \mathrm{MS}$ in cyclohexane at $30.5^{\circ} \mathrm{C}(\Theta)$. The solid and dashed curves represent the theoretical values for the HW model and the rigid sphere model, respectively. the one $8 \times 10^{3} \AA^{3}$ for i-PMMA. ${ }^{12}$

\section{DISCUSSION}

\section{HW Theory}

In order to make a comparison of the present results of depolarized LS measurements with the (dynamic) HW theory, ${ }^{1,13}$ it is convenient to begin by giving its brief explanation. The model used is the discrete HW chain ${ }^{1,20}$ such that it is composed of $N$ identical rigid subbodies, each with the translational and rotatory friction coefficients $\zeta_{\mathrm{t}}$ and $\zeta_{\mathrm{r}}$, joined successively with bonds of fixed length $a$, and that the orientation of each subbody with respect to its predecessor is described by the potential energy of the original continuous HW chain. Therefore, the basic model parameters for the former are the same as those for the latter, i.e., the constant differential-geometrical curvature $\kappa_{0}$ and torsion $\tau_{0}$ of the characteristic helix of the latter taken at the minimum zero of its elastic energy and the static stiffness parameter $\lambda^{-1}$.

For the single discrete HW chain so defined, the power spectrum $J_{\Gamma}(\Delta \omega)$ of the depolarized component of the light scattered from it as a function of the difference $\Delta \omega$ between the angular frequencies of the scattered and incident light waves may be written in the form ${ }^{1,13}$

$$
J_{\Gamma}(\Delta \omega)=\sum_{k \text { odd }} \sum_{j=-2}^{2} A_{k}^{j} \frac{\tau_{2, k}^{j}}{1+\left(\Delta \omega \tau_{2, k}^{j}\right)^{2}}
$$

where

$$
\begin{gathered}
\tau_{2, k}^{j}=1 / \lambda_{2, k}^{j} \\
A_{k}^{j}=\frac{12 \pi}{(N+1)} B_{k}^{j} \cot ^{2}\left[\frac{\pi k}{2(N+1)}\right]
\end{gathered}
$$

with

$$
B_{k}^{j}=\left(8 \pi^{2}\right)^{-1}\left|\sum_{j^{\prime}=-2}^{2} \alpha_{2}^{j^{\prime}} R_{2, k}^{j j^{\prime}}\right|^{2}
$$

In eq $2, \lambda_{2, k}^{j}$ are the eigenvalues of the matrix representation of the diffusion operator associated with the subspace (of full Hilbert space) spanned by the basis set with the "total angular momentum quantum number" $L=2$ and the number of "excited" subbodies $n=1$. Specifically, $J_{\Gamma}(\Delta \omega)$ is the Fourier-Laplace transform of a linear combination of the basic timecorrelation functions associated with that subspace. We use the augmented eigenvalues $\lambda_{2, k}^{j}$ given by eq 25 of ref 15 (or eq 49 of ref 13) which takes partly into account the interactions with the complementary subspace. Thus $\lambda_{2, k}^{j}$ may readily be calculated for given values of $N$ and the six model parameters introduced above: $\kappa_{0}, \tau_{0}, \lambda^{-1}, a, \zeta_{\mathrm{t}}$, and $\zeta_{\mathrm{r}}$. (Of course, 
the absolute temperature $T$ should also be specified.) In eq $4, \alpha_{2}^{j}$ are the spherical tensor components of the polarizability tensor affixed to the subbody, and $R_{2, k}^{j j^{\prime}}$ is the transformation matrix associated with the eigenvalue problem of the above-mentioned matrix and is given by eq 40 of ref 13 . We note that $R_{2, k}^{j j^{\prime}}$ depends on the parameters $\kappa_{0}, \tau_{0}$, and $\lambda^{-1}$. As explicitly shown in the previous theoretical paper, ${ }^{13}$ Lorentzians only with very small $k$ make contribution to $J_{\Gamma}$ because of the factor $\cot ^{2}[\cdots]$ involved in the amplitude $A_{k}^{j}$ given by eq 3 , and moreover, only those belonging to one or two branches $(j)$ of the eigenvalue spectrum $\left(\lambda_{2, k}^{j}\right)$ make contribution because of the factor $B_{k}^{j}$ given by eq 4 . The resultant $J_{\Gamma}$ may therefore be represented practically in terms of a single Lorentzian. This is consistent with the previous and present experimental results.

\section{Dependence of $\tau_{\Gamma}$ on $x_{w}$}

Now we proceed to make a comparison of the present data for $\tau_{\Gamma}$ with the HW theory. In Figure 2, the solid curve represents the HW theoretical values of $\tau_{\Gamma}$ given as the reciprocal of the HWHM of $J_{\Gamma}$ calculated from eqs 1 and 2 with the values of the (static) model parameters determined from $\left\langle S^{2}\right\rangle,{ }^{3}$ i.e., $\lambda^{-1} \kappa_{0}=3.0, \lambda^{-1} \tau_{0}=0.9, \lambda^{-1}=46.8 \AA$, and $\lambda a=$ $0.063(a=2.95 \AA)$, those of the (dynamic) parameters, i.e., $r_{1} \equiv \zeta_{\mathrm{t}} / 3 \pi \eta_{0} a=1.0$ and $r_{2} \equiv \zeta_{\mathrm{r}} / a^{2} \zeta_{\mathrm{t}}=$ 8.0, and those of the components of the polarizability tensor $\boldsymbol{\alpha}$ of the subbody (repeat unit) expressed in the localized Cartesian coordinate system affixed to it, which are the same as those previously ${ }^{4}$ used to calculate $\left\langle\Gamma^{2}\right\rangle$ and are given by

$$
\boldsymbol{\alpha}=\operatorname{diag}(1.40,2.47,-3.87) \AA^{3} \quad(\mathrm{a}-\mathrm{P} \alpha \mathrm{MS})
$$

where $N$ has been set equal to $x_{\mathrm{w}}$. Recall that $\left\langle\Gamma^{2}\right\rangle$ is equal to the integral of $J_{\Gamma}$ over the whole range of $\Delta \omega$ at zero scattering angle. ${ }^{1,13}$

We note that the above value of $\lambda a$ has been calculated from eq 9.14 of ref 1 (or eq 22 of ref 20) with the value 0.0634 of $\lambda \Delta s=\lambda M_{0} / M_{\mathrm{L}}$, where $M_{0}$ is the molecular weight of the repeat unit and is taken to be 118 for a-P $\alpha \mathrm{MS}$ and $M_{\mathrm{L}}$ is the shift factor as defined as the molecular weight per unit contour length and has been determined to be $39.8 \AA^{-1}$ for a-P $\alpha \mathrm{MS} .^{3}$ We also note that the above values of $r_{1}$ and $r_{2}$ are the same as those previously used for a-PS ${ }^{10}$ and a- and iPMMAs. ${ }^{11,12}$ If we assume as in the previous studies $^{10-12}$ that the subbody is an oblate spheroid having the rotation axis of length $a$ and the diameter $d$, then $d$ is calculated to be $11 \AA$ from eq 10.18 with eqs 10.19 , 6.A.47, and 6.A.48 of ref 1 (or eqs 35-38 of ref 21) with the above values of $a, r_{1}$, and $r_{2}$. This value of $d$ is in good agreement with the value $11 \AA$ previous$1 y^{15}$ estimated from the chemical structure of syndio- tactic $\mathrm{P} \alpha \mathrm{MS}$ (with $f_{\mathrm{r}}=1.0$ ), indicating that the above values of $r_{1}$ and $r_{2}$ are reasonable.

In Figure 2, we have omitted the HW theoretical values for $x_{\mathrm{w}} \lesssim 10$ since the block-diagonal approximation $^{1,22}$ adopted in that theory breaks down in the range of such small $N$. The theoretical asymptotic value of $k_{\mathrm{B}} T \tau_{\Gamma} / \eta_{0}$ in the limit of $x_{\mathrm{W}} \rightarrow \infty$ is $2.07 \times$ $10^{4} \AA^{3}$ and is ca. 2.4 times as large as the experimental value $c a .8 .5 \times 10^{3} \AA^{3}$. As in the cases of a- and iPMMAs, ${ }^{11,12}$ agreement between theory and experiment is only semiquantitative and is not so good as in the case of a-PS. ${ }^{10}$

As in the previous studies of a-PS ${ }^{10}$ and a- and iPMMAs, ${ }^{11,12}$ we consider $\tau_{\Gamma}$ for the rigid sphere mod$\mathrm{el}^{1}$ having the radius equal to the apparent root-meansquare radius of gyration $\left\langle S^{2}\right\rangle_{\mathrm{s}}^{1 / 2}$ of the HW chain and having a cylindrically symmetric polarizability tensor. It may be given by

$$
\tau_{\Gamma}=4 \pi \eta_{0}\left\langle S^{2}\right\rangle_{\mathrm{s}}^{3 / 2} / 3 k_{\mathrm{B}} T
$$

We note that $\left\langle S^{2}\right\rangle_{\mathrm{s}}^{1 / 2}$ is defined as the coefficient of the squared scattering vector $k^{2}$ in the expansion of the scattering function determined from small-angle $\mathrm{X}$ ray scattering measurements and may be regarded as the mean-square radius of gyration of the excess electron density. Thus it may be given by

$$
\left\langle S^{2}\right\rangle_{\mathrm{s}}=\left\langle S^{2}\right\rangle+S_{\mathrm{c}}^{2}
$$

where $\left\langle S^{2}\right\rangle$ is the (usual) mean-square radius of gyration of the HW chain contour of total length $L$ and is given by eq 5 of ref 3 (or eq 4.83 of ref 1 in units of $\lambda^{-1}$ ) and $S_{\mathrm{c}}$ is the (effective) radius of gyration of the cross section of the excess electron density distributed around the chain contour and has already been evaluated to be $\sqrt{10.7} \AA$ for a-P $\alpha \mathrm{MS}^{3}{ }^{3}$ The values of $\tau_{\Gamma}$ calculated from eq 6 with eq 7 are represented by the dashed curve in Figure 2. We note that the contour length $L$ of the HW chain has been converted to $x_{\mathrm{w}}$ by the use of the relation $x_{\mathrm{w}}=M_{\mathrm{L}} L / M_{0}$. The dashed curve may reproduce satisfactorily the data points for $x_{\mathrm{w}} \lesssim 10$ as in the cases of a-PS ${ }^{10}$ and a- and iPMMAs. ${ }^{11,12}$

\section{Comparison with Other Flexible Polymers}

Finally, in this subsection we compare the present results for a-P $\alpha \mathrm{MS}$ with the previous ones for a-PS ${ }^{10}$ and a- and i-PMMAs. ${ }^{11,12}$ Figure 3 shows doublelogarithmic plots of $\tau_{\Gamma} / \tau_{\Gamma}^{0}$ against $x_{\mathrm{w}}$ for a-P $\alpha \mathrm{MS}$ in cyclohexane at $30.5^{\circ} \mathrm{C}(\Theta)$ (unfilled circles), a-PS in cyclohexane at $34.5^{\circ} \mathrm{C}(\Theta)$ (filled circles), a-PMMA in acetonitrile at $44.0^{\circ} \mathrm{C}(\Theta)$ (filled triangles), and iPMMA in acetonitrile at $28.0^{\circ} \mathrm{C}(\Theta)$ (filled inverted triangles), where $\tau_{\Gamma}^{0}$ is the $\tau_{\Gamma}$ of the monomer (isolated repeat unit), i.e., $\tau_{\Gamma}^{0}=0.0060_{7} \mathrm{~ns}$ for a-P $\alpha \mathrm{MS}$ (cumene) in cyclohexane at $30.5^{\circ} \mathrm{C}, 0.0056_{2} \mathrm{~ns}$ for a- 


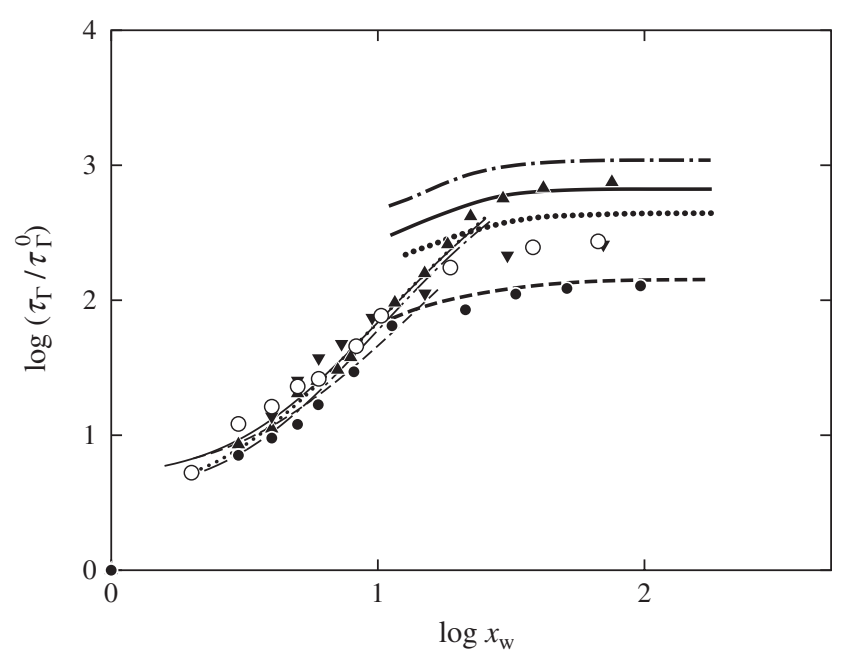

Figure 3. Double-logarithmic plots of $\tau_{\Gamma} / \tau_{\Gamma}^{0}$ against $x_{\mathrm{w}}$, where $\tau_{\Gamma}^{0}$ is the $\tau_{\Gamma}$ of the isolated monomer: (O) present data for a-P $\alpha \mathrm{MS}$ in cyclohexane at $30.5^{\circ} \mathrm{C}(\Theta) ;(\bullet)$ previous data for a-PS in cyclohexane at $34.5^{\circ} \mathrm{C}(\Theta) ;{ }^{10}(\mathbf{\Delta})$ previous data for a-PMMA in acetonitrile at $44.0^{\circ} \mathrm{C}(\Theta) ;{ }^{11}(\boldsymbol{\nabla})$ previous data for i-PMMA in acetonitrile at $28.0^{\circ} \mathrm{C}(\Theta) .{ }^{12}$ The heavy solid, dashed, dot-dashed, and dotted curves represent the respective HW theoretical values, and the light curves represent the respective theoretical values for the rigid sphere model.

PS (cumene) in cyclohexane at $34.5^{\circ} \mathrm{C},{ }^{10} 0.0019_{3} \mathrm{~ns}$ for a-PMMA (methyl isobutylate) in acetonitrile at $44.0{ }^{\circ} \mathrm{C},{ }^{11}$ and $0.0023_{6}$ ns for i-PMMA (methyl isobutylate) in acetonitrile at $28.0^{\circ} \mathrm{C} .{ }^{12}$ The above value $0.0060_{7} \mathrm{~ns}$ of $\tau_{\Gamma}^{0}$ in cyclohexane at $30.5^{\circ} \mathrm{C}$ has been calculated from the value $0.0056_{2}$ ns previously ${ }^{10}$ determined in cyclohexane at $34.5^{\circ} \mathrm{C}$ by assuming that the reduced relaxation time $k_{\mathrm{B}} T \tau_{\Gamma}^{0} / \eta_{0}$ is independent of $T$. As previously noted, ${ }^{11,12}$ the values of the reduced relaxation time $k_{\mathrm{B}} T \tau_{\Gamma}^{0} / \eta_{0}$ are $31 .{ }_{1}$ and $29 .{ }_{6} \AA^{3}$ for cumene and methyl isobutylate, respectively, and are in good agreement with each other.

The asymptotic value $\tau_{\Gamma, \infty} / \tau_{\Gamma}^{0}$ of the ratio $\tau_{\Gamma} / \tau_{\Gamma}^{0}$ in the limit of $x_{\mathrm{w}} \rightarrow \infty$ represents the degree of restriction on the rotational motion of the isolated motional unit (monomer) due to its incorporation into the long enough polymer chain and may be regarded as a measure of "dynamic chain stiffness" (associated with $\left.J_{\Gamma}\right){ }^{1,15}$ The heavy solid, dashed, dot-dashed, and dotted curves represent the HW theoretical values of $\tau_{\Gamma}$ for a-P $\alpha \mathrm{MS}$, a-PS, a- and i-PMMAs, respectively, divided by the respective observed ones of $\tau_{\Gamma}^{0}$, and the light curves represent the respective theoretical values for the rigid sphere model.

Although the values of the ratio $\tau_{\Gamma} / \tau_{\Gamma}^{0}$ for a-P $\alpha \mathrm{MS}$, a-PS, a- and i-PMMAs are close to each other in the oligomer region with very small $x_{\mathrm{w}}$, they deviate from each other with increasing $x_{\mathrm{w}}$. In the limit of $x_{\mathrm{w}} \rightarrow \infty$, the value of the ratio for a-P $\alpha \mathrm{MS}$ is intermediate between those for a-PMMA and a-PS, and is slightly larger than that for i-PMMA, reconfirming our previous conclusion ${ }^{1,15}$ that there is good correlation between the dynamic and static chain stiffness. We note that the values of $\lambda^{-1}$ are $20.6 \AA$ (a-PS) $<38.0 \AA$ (iPMMA) $<46.8 \AA$ (a-P $\alpha$ MS) $<57.9 \AA$ (a-PMMA). ${ }^{1}$ Considering the fact that the HW theoretical values have been calculated by the use of the values of the HW model parameters determined from the equilibrium property $\left\langle S^{2}\right\rangle$ along with the proper values of the dynamic parameters, it may be concluded that the dynamic HW theory may explain well the difference in $\tau_{\Gamma, \infty} / \tau_{\Gamma}^{0}$ among a-P $\alpha$ MS, a-PS, a- and i-PMMAs, although agreement between theory and experiment is only semiquantitative except for a-PS.

\section{CONCLUDING REMARKS}

The power spectra $J_{\Gamma}$ of the excess depolarized component of the light scattered from a-P $\alpha \mathrm{MS}$ in cyclohexane at $30.5^{\circ} \mathrm{C}(\Theta)$ were determined from frequency-domain dynamic depolarized LS measurements following the data-processing procedure previously developed in order to extract $J_{\Gamma}$ from observed spectra. Then it has been found that $J_{\Gamma}$ may be well represented in terms of a single Lorentzian independently of $x_{\mathrm{w}}$, and that the relaxation time $\tau_{\Gamma}$ defined by the reciprocal of the HWHM of $J_{\Gamma}$ increases with increasing $x_{\mathrm{w}}$ and levels off to its asymptotic value in the limit of $x_{\mathrm{w}} \rightarrow \infty$ as in the previous cases of a-PS ${ }^{10}$ and a- and i-PMMAs, ${ }^{11,12}$ being consistent with the theoretical prediction on the basis of the HW chain model. From a rather detailed analysis of the present data, it has been shown that the HW theory may explain semiquantitatively the observed dependence of $\tau_{\Gamma}$ (or $J_{\Gamma}$ ) on $x_{\mathrm{w}}$ over that range of $x_{\mathrm{w}}$ for which the theory is applicable, i.e., for $x_{\mathrm{w}} \gtrsim 10$, while the rigid sphere model ${ }^{1,10-12}$ having the radius equal to the apparent root-mean-square radius of gyration $\left\langle S^{2}\right\rangle_{\mathrm{s}}^{1 / 2}$ of the HW chain may reproduce well the data for $x_{\mathrm{w}} \lesssim 10$. From a comparison of the present results for a-P $\alpha$ MS with the previous ones for a-PS and a- and i-PMMAs, it has also been shown that the dynamic chain stiffness ${ }^{1}$ defined as the ratio of the relaxation time associated with the local motion of the long chain to that of its isolated repeat unit (monomer) is well correlated with the static chain stiffness $\lambda^{-1}$, as predicted by the HW theory.

In the following paper, ${ }^{9}$ we make a nuclear magnetic relaxation study of a-P $\alpha$ MS in a similar spirit.

Acknowledgment. This research was supported in part by the 21st century COE program "COE for a United Approach to New Materials Science" from the Ministry of Education, Culture, Sports, Science, and Technology, Japan. 


\section{REFERENCES}

1. H. Yamakawa, "Helical Wormlike Chains in Polymer Solutions," Springer, Berlin, 1997.

2. H. Yamakawa, Polym. J., 31, 109 (1999).

3. M. Osa, T. Yoshizaki, and H. Yamakawa, Macromolecules, 33, 4828 (2000).

4. H. Kojo, M. Osa, T. Yoshizaki, and H. Yamakawa, Macromolecules, 36, 6570 (2003).

5. Y. Ohgaru, M. Sumida, M. Osa, T. Yoshizaki, and H. Yamakawa, Macromolecules, 33, 9316 (2000).

6. I. Suda, Y. Tominaga, M. Osa, T. Yoshizaki, and H. Yamakawa, Macromolecules, 33, 9322 (2000).

7. M. Osa, M. Sumida, T. Yoshizaki, H. Yamakawa, K. Ute, T. Kitayama, and K. Hatada, Polym. J., 32, 361 (2000).

8. Y. Tamai, T. Konishi, Y. Einaga, M. Fujii, and $\mathrm{H}$. Yamakawa, Macromolecules, 23, 4067 (1990).

9. M. Osa, H. Ueda, T. Yoshizaki, and H. Yamakawa, Polym. J., 37, 14 (2005).

10. Y. Takaeda, T. Yoshizaki, and H. Yamakawa, Macromolecules, 27, 4248 (1994).

11. Y. Takaeda, T. Yoshizaki, and H. Yamakawa, Macromole- cules, 28, 682 (1995).

12. Y. Naito, N. Sawatari, Y. Takaeda, T. Yoshizaki, and H. Yamakawa, Macromolecules, 30, 2751 (1997).

13. T. Yoshizaki and H. Yamakawa, J. Chem. Phys., 99, 9145 (1993).

14. H. Yamakawa and M. Fujii, J. Chem. Phys., 81, 997 (1984).

15. H. Yamakawa, T. Yoshizaki, and M. Fujii, J. Chem. Phys., 84, 4693 (1986).

16. Y. Takaeda, T. Yoshizaki, and H. Yamakawa, Macromolecules, 26, 3742 (1993).

17. Y. Takaeda, T. Yoshizaki, and H. Yamakawa, Macromolecules, 28, 4167 (1995).

18. A. C. Ouano and R. Pecora, Macromolecules, 13, 1167 (1980).

19. T. Yoshizaki, Y. Takaeda, and H. Yamakawa, Macromolecules, 26, 6891 (1993).

20. H. Yamakawa and T. Yoshizaki, J. Chem. Phys., 75, 1016 (1981).

21. T. Yoshizaki and H. Yamakawa, J. Chem. Phys., 81, 982 (1984).

22. H. Yamakawa, T. Yoshizaki, and J. Shimada, J. Chem. Phys., 78, 560 (1983). 